\title{
Mycoplasma bovis infection in dairy herds-Risk factors and effect of control measures
}

\author{
Vera Haapala, ${ }^{1 *}$ ๑ Nella Vähänikkilä, ${ }^{2}$ @ Laura Kulkas, ${ }^{3}$ Erja Tuunainen, ${ }^{4}$ Tarja Pohjanvirta, ${ }^{2}$ Tiina Autio, ${ }^{2}$ () \\ Sinikka Pelkonen, ${ }^{2}$ Timo Soveri, ${ }^{1}$ and Heli Simojoki ${ }^{1,5}$ (]) \\ ${ }^{1}$ Department of Production Animal Medicine, Faculty of Veterinary Medicine, University of Helsinki, Paroninkuja 20, 04920 Saarentaus, Finland \\ ${ }^{2}$ Finnish Food Authority, Neulaniementie 4, 70210 Kuopio, Finland \\ ${ }^{3}$ Valio Oy, Meijeritie 6, 00370 Helsinki, Finland \\ ${ }^{4}$ Animal Health ETT ry, PL 221, 60101 Seinäjoki, Finland \\ ${ }^{5}$ Department of Agricultural Sciences, University of Helsinki, PL 27 00014, Helsinki, Finland
}

\section{ABSTRACT}

As Mycoplasma bovis spreads to new countries and becomes increasingly recognized as a disease with major welfare and economic effects, control measures on dairy farms are needed. To minimize the risk of infection spread to naive herds, all possible risk factors for $M$. bovis infection should be identified and controlled. Mycoplasma bovis was first diagnosed in dairy cattle in Finland in 2012, and by January 2020, 86 Finnish dairy farms $(<1.5 \%)$ supporting $M$. bovis infections were identified. We evaluated risk factors for $M$. bovis infection using a questionnaire provided to 40 infected and 30 control dairy farms. Control measures were advised for 19 of the infected dairy farms during visits by a veterinarian. The course of the infection on those farms was followed by analyzing calf nasal swabs with PCR for presence of $M$. bovis 4 times at 6-mo intervals. Control measures included culling of $M$. bovis mastitic cows, isolation of new calves from older animals after initial $M$. bovis mastitic cows had been culled, prevention of nose-to-nose contact with infected animals, early detection of mastitis cases using $M$. bovis PCR, and hygiene measures mainly related to milking, calf pens, feeding buckets, and teats. Farms implemented the control measures related to the isolation of calves or avoidance of nose-to-nose contact in various ways, according to farm structures and financial circumstances. In our study, the control measures recommended to the dairy farms appeared effective, such that 13 of 19 farms reached a low risk level during at least 3 consecutive negative samplings from calves, with no $M$. bovis mastitis detected subsequently. Among risk factors, insemina-

Received April 30, 2020.

Accepted September 11, 2020.

*Corresponding author: vera.haapala@helsinki.fi tion with an $M$. bovis-positive bull indicated a trend of increasing the odds of $M$. bovis infection on the farm in a multivariable logistic model. In contrast, higher herd average milk yield had an association with lower odds for $M$. bovis infection. Occurrence of other infectious diseases affecting several animals on the dairy farm in the previous 6 mo before $M$. bovis infection were more frequent on $M$. bovis-infected farms.

Key words: control measure, dairy cattle, Mycoplasma bovis, risk factor

\section{INTRODUCTION}

Mycoplasma bovis causes various diseases, including mastitis, arthritis, and pneumonia (Maunsell et al., 2011). In addition, the efficacy of antibiotic treatment is poor (Gautier-Bouchardon et al., 2014), and diagnosing asymptomatic carrier animals is difficult (Maunsell et al., 2011). Therefore, dairy farms would benefit from effective control measures. Recent studies identified purchased cattle (Schibrowski et al., 2018; Murai and Higuchi, 2019; Fujimoto et al., 2020) and the use of a breeding bull (Gille et al., 2018) or contaminated semen used in insemination (Haapala et al., 2018) as external risk factors for the occurrence of $M$. bovis in dairy herds.

Mycoplasma bovis has spread to several countries (Nicholas and Ayling, 2003) after it was first isolated in 1961 in the United States (Hale et al., 1962). As we described in Vähänikkilä et al. (2019), M. bovis quickly spreads to all age groups once it enters a herd. Aebi et al. (2015) reported a prolonged colonization in clinically healthy animals, concluding that young stock should be taken into account as a reservoir. Timsit et al. (2012) reported horizontal transmission from older animals into adjacent pens. Based on this knowledge, newborn calves can get the infection from older animals and maintain the chain of infection in the herd. Calves at the age of 1 to 4 mo have the highest prevalence of 
positive nasal swabs, and the prevalence drops at the age of 8 to 12 mo (Bennett and Jasper, 1977). The key to stopping the chain of infection is to stop the spread to new calves born after the detection of $M$. bovis on the farm.

Actions for controlling the infection presented in the literature are culling or isolating $M$. bovis mastitic cows (Bicknell et al., 1983; Byrne et al., 1998; Punyapornwithaya et al., 2012), pasteurization of infected milk (Butler et al., 2000), raising the calves separately from older animals (Brys et al., 1992; Pfützner and Sachse, 1996; Aebi et al., 2015), better milking hygiene (Bicknell et al., 1983; Punyapornwithaya et al., 2012), teat dipping (Boddie et al., 2002), and in extreme cases, culling all cattle, including young stock (Pothmann et al., 2015). Basic principles for disease control, such as the use of hospital pens for diseased animals, improved hygiene and ventilation, and avoidance of overcrowding, are also important (Gorden and Plummer, 2010).

New Zealand attempts to eradicate $M$. bovis with intensive testing to detect all $M$. bovis-positive farms and culling all the cattle on infected farms (Biosecurity New Zealand, 2020). As far as we know, other countries try to control the infection on farms mainly by isolating or culling the $M$. bovis mastitis cases and placing animals with pneumonia or arthritis in hospital pens. If the infection could be eradicated from dairy farms, it would reduce costs, labor, and the use of antibiotics and promote animal welfare. To our knowledge, only few studies have been published on eradication of $M$. bovis, and they concentrate on either eradicating $M$. bovis mastitis (Bicknell et al., 1983; Byrne et al., 1998; Punyapornwithaya et al., 2012) or raising M. bovis-free calves (Brys et al., 1992). In a review, Pfützner and Sachse (1996) mentioned that eradication of $M$. bovis is possible by keeping the noninfected animals away from the infected cattle their whole life, but concluded that this is expensive and laborious. The aims of this study were to identify risk factors for $M$. bovis infection at the herd level and to determine if control measures implemented on $M$. bovis-infected farms help the farm to reach a low risk level of $M$. bovis infection.

\section{MATERIALS AND METHODS}

Risk factors for the introduction of $M$. bovis infection were analyzed with an observational case-control study. The effect of control measures in dairy farms were analyzed with an observational prospective cohort study. In a study of Vähänikkilä et al. (2019), we concentrated on the course of $M$. bovis infection on these dairy farms and the best diagnostic tools. In this paper, in addition to risk factors, we concentrate on the effects of control measures in the study farms.

\section{Mycoplasma bovis in Finland}

Mycoplasma bovis was detected in Finland in 2012. In 2013, a voluntary $M$. bovis control program was established by Animal Health ETT (Animal Health ETT, Seinäjoki, Finland) to decrease the risk of introducing the infection related to animal purchase. In the program, farms are categorized according to their M. bovis status, based mainly on several consecutive nasal swab (NS) samples from calves, surveillance of clinical signs in regular herd health visits, bulk tank milk (BTM) samples taken twice a year, and routine testing of mastitis milk samples for $M$. bovis with PCR. Categories in the program are A-level and joining level. Purchase of cattle for farms in the program is allowed only from the A-level, and health certificates are used when purchasing cattle or attending animal fairs. Farms in the program have a disease-prevention plan designed by the herd veterinarian. Additional farm categories are low risk level, $M$. bovis status unknown, $M$. bovis suspected, and $M$. bovis positive. Negative farms are at the highest A-level after an minimum 8-mo follow-up period, including 2 herd health visits with negative NS and milk samples (individual and tank milk). Mycoplasma bovispositive farms are encouraged to aim for low risk level, which they can achieve with 3 consecutive negative NS samples (10-20), negative milk samples analyzed for $M$. bovis with PCR, and surveillance of signs.

\section{Case-Control Study for Risk Factors}

Case-Control Study Farms. The case farms included 40 recently discovered $M$. bovis-infected dairy herds, accounting for almost $70 \%$ of the infected farms at that time. All M. bovis-positive dairy farms in Finland that were willing to participate and filled in the questionnaire were included. Nineteen of the farms were among the first dairy farms identified as having $M$. bovis after the infection was detected in Finland. We described the course of $M$. bovis infection in those 19 herds recently (Vähänikkilä et al., 2019).

Control farms $(\mathrm{n}=30)$ were matched according to the geographical location with infected case farms. Local veterinarians were asked if they had clients that would be willing to participate. In addition, control farms were similar to the case farms with respect to barn type (loosestall, tiestall). The farms had routinely been tested, with negative results, for quarter milk samples (QMS) from clinical and subclinical mastitis cases in commercial laboratories using a mastitis pathogen PCR kit including $M$. bovis (Pathoproof Complete 16-kit, Thermo Fisher Scientific, Vantaa, Finland).

Data. Risk factor data collection was done with an online questionnaire, including for several internal and 
external biosecurity issues on the farm (Table 1). A veterinarian visited the case farms and either the veterinarian (by interviewing the farmer) or the farmer filled out the questionnaire. Farmers of control farms completed the online questionnaire. For all farms, the data for the numbers of purchased cattle in recent years, herd size, and milk yield were obtained from the centralized na- tional health care register Naseva (https://www.naseva .fi/PublicContent/IntroductionInEnglish), maintained by Animal Health ETT. Data for inseminations using semen from a bull infected with $M$. bovis were collected from the database of the Faba co-op breeding company (Vantaa, Finland). Case farms had a few additional questions related to the $M$. bovis infection on the farm

Table 1. Description of the questions asked in the online questionnaire of the case and control farms to evaluate the external risk factors for a herd to get Mycoplasma bovis infection and internal factors possibly affecting the spread of infection among animal groups. Reply options to questions were yes or no if other options are not mentioned

Subject Description

M. bovis status

Farm details

Animal movements

Visitors

Calves

Signs

Mastitis detection

Other infectious diseases

Obtained from the national centralized health care register Naseva ${ }^{3}$

Only for the case farms
Date when $M$. bovis was detected on the farm, if detected

Stall system (loosestall, tiestall, other), milking system (parlor, pipeline, $\mathrm{AMS}^{1}$ ), number of cows, number of cows has increased significantly (yes in the last year, yes within 3 years, yes over 3 years ago, no), mean herd milk yield from a previous year before the infection was detected

Contract heifer unit used, purchased animals, ${ }^{2}$ imported animals,${ }^{2}$ imported embryos, ${ }^{2}$ domestic embryos, ${ }^{2}$ animals attended animal fairs or other farms or places with cattle, ${ }^{2}$ cattle from other farms have visited ${ }^{2}$

Hygienic lock by the door, boots provided (yes always or usually, no), protective coat or overalls provided (yes always or usually, no), veterinarian always uses boots and coat or overall provided (yes, no), inseminator always uses boots and apron or overall provided (yes, no, farmer inseminates), hoof trimmer uses always boots and coat or overall provided (yes, no, not valid), AMS maintenance always uses the boots and coat and overall provided (yes, no, not valid), other visitors use boots and overall or coat provided (yes, no), farmers move the animals sold out of the barn themselves (yes, usually, no)

The length of newborn calves kept with the dam (removed immediately after calving, few hours dam licks the calf, few days, more than few days), colostrum from other farms, high cell count milk fed to calves without pasteurization, all-in-all-out system used with calves, milk-fed calves in separate area (yes and separate air ventilation, yes and incoming air from the area with other animals, only youngest milk-fed calves and separate air ventilation, only youngest milk-fed calves and incoming air from the area with other animals, no), number of milk-fed calf groups $(1,2$, over 2 ), rearing of farm's own bull calves for meat (yes until slaughter, yes until 3-6 mo, no, other)

Signs related to $M$. bovis listed and asked if these have occurred on the farm in the last $3 \mathrm{yr}$ [no, yes (several or individual), and maybe (needs to be explained)]; signs listed in all animal groups (bulls, cows, heifers, young stock 6-22 mo, and calves) were respiratory, eye, and jointrelated signs; in addition, related to young stock and calves, ear drooping, ear infection, and neurological signs were listed.

Is bacteriological analysis done to the mastitis cases (always, often, randomly, no)? What method is used to analyze mastitis samples (PCR, culture not detecting $M$. bovis, culture by laboratory, not analyzed, several methods used clarify)? If PCR is not used in mastitis diagnosis, has there been mastitis cases not responding to antibiotic treatment (yes several, yes few, no, not valid)? If PCR is not used in mastitis diagnosis has the number of high cell count cows increased (yes, no, not valid)?

Has other infectious disease been on the farm in the last $6 \mathrm{mo}$, for example, rotavirus, interdigital phlegmon, ringworm, respiratory diseases, and Streptococcus agalactiae mastitis (yes in several animals, yes in few animals, no)?

Amount of purchased cattle in the last $3 \mathrm{yr}$, number of cows, milk yield

How many cows have had $M$. bovis mastitis? How many mastitis cows had signs (general or udder) at the time of sampling? How many mastitis cows only had high cell count and the milk looked normal? In how many M. bovis mastitis cases the cow had no signs and the cell count was normal? Has the $M$. bovis mastitis cow been culled [yes all of them, yes part of them (how many), no]? Where was $M$. bovis infection first detected (mastitis, respiratory signs, other)? Has the slaughter house informed of an increase of lung or joint lesions in meat inspection? Has the amount of medication used increased in the last 3 yr related to signs of M. bovis other than mastitis [respiratory infections, arthritis, otitis, eye infections, neurological signs (yes clearly, yes some, no, less)]?

\footnotetext{
${ }^{1} \mathrm{AMS}=$ automated milking system.

${ }^{2}$ Within 3 yr before detecting $M$. bovis the first time.

${ }^{3}$ Administered by ETT Animal Health (Seinäjoki, Finland).
} 
Table 2. Control measures evaluated against Mycoplasma bovis on 19 follow-up dairy farms

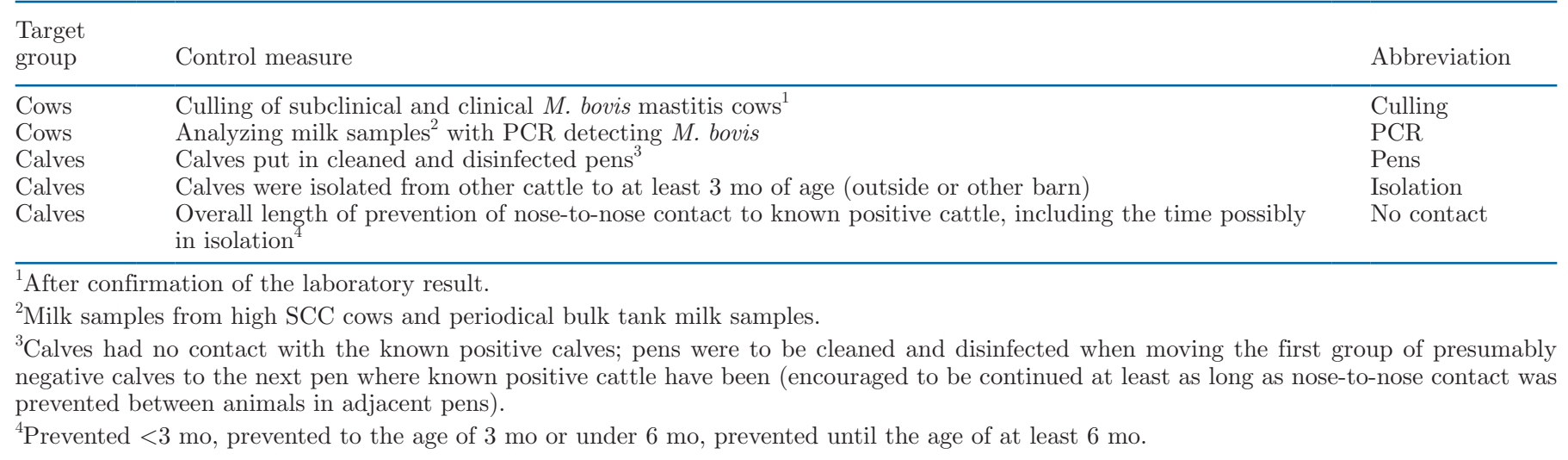

(Table 1). The farmers gave consent to use their data in research.

\section{Effect of Control Measures on M. bovis Infection}

Follow-Up Farms. The effect of control measures was studied on 19 farms where the course of $M$. bovis infection was followed as we described in Vähänikkilä et al. (2019). Those farmers filled in the questionnaire intended for the case farms in the case-control study. In addition, we recorded the purchase of new animals during sampling, which influences the efficacy of the control measures.

Control Measures. Farmers received advice as soon as possible after the initial detection of $M$. bovis on the farms. We advised the farmers to avoid purchasing new animals, to cull cows with $M$. bovis mastitis, to house calves separately from the older animals, and to follow specific hygiene measures. The control measures included in the study are listed in Table 2.

Control measures were based on the need to keep the calves born after the detection of $M$. bovis away from the older animals, so that the new calves would not become infected. This is based on the hypothesis that older animals will eventually be cleared of $M$. bovis if these animals do not get a new infection. Isolation of calves or preventing nose-to-nose contact with older animals was started after the initial $M$. bovis mastitis cases had been detected and those cows culled.

Isolation of calves is described as a situation where calves born from that time on are raised separately from the older animals. These isolated calves were kept in a different air space, for example, outside in hutches, temporary pens, or in a different barn. Isolated calves were kept in various sized groups, first in individual or group pens and then in small groups of approximately 4 to 6 calves. It was recommended that the calves be isolated for at least 6 mo. After moving the animals back into the barn, it was advised to prevent nose-to-nose contact with the assumed-positive animals for as long as possible, ideally until the heifers were transferred to join the milking cows. If isolation was not carried out and the newborn calves remained in the same air space as the animals presumed to be $M$. bovis positive, nose-to-nose contact of the newly born calves and older animals was prevented. This was done in the group pens by placing high wooden boards between pens if calves and older animals were next to each other. If pens had common water sources, animal in those pens were considered as having nose-to-nose contact. Farms had different-sized group pens, usually 5 to 10 animals but also about 30 animals in 1 farm.

In addition to control measures included in the study, the farmer was advised to segregate (e.g., place in a sick pen) $M$. bovis mastitic cows and milk them separately until culling. It was advised to analyze BTM samples for $M$. bovis with PCR once a month for the duration of the project and more frequently at the time of the $M$. bovis mastitis infection. This was done to enhance the probability of detecting possible subclinical M. bovispositive mastitic cows. During the time the infection was actively spreading among the cows, it was advised that the calves be moved immediately after parturition, before the dam licked the newborn. General hygiene instructions were given related to milking, milk feeding buckets, automatic feeding teats, young stock, and hospital pens. When the acute phase lingered among the cows with several mastitis cases or signs of pneumonia, we instructed disinfection of drinking fountains, feeding tables (if possible), and bowls after culling the last cow with $M$. bovis mastitis and after severe respiratory signs had been resolved. Farmers were instructed not to feed colostrum to the calves from cows with high SCC milk. In addition, it was instructed that separated high SCC milk was not to be fed to the calves unless the milk was pasteurized. It was mentioned in the instruction that 
the use of milk replacers could be considered, but the use of them was not specifically monitored.

It was instructed that high SCC milk should be analyzed for M. bovis with PCR. After calving, it was advised that the cows were screened for 2 weeks with a California Mastitis Test until all the cows that were dried off at the time of detection of the $M$. bovis on the farm had calved. To reach a low risk status, an infected farm was required to have 3 consecutive PCR negative NS samples (4-8 mo apart) from 10 to 20 calves and negative PCR results for clinical QMS and BTM samples. The number of BTM samples taken in each farm during the project are described in Vähänikkilä et al. (2019). Some farms also took BTM samples during the follow-up period but the amount and interval of these was not constant. A veterinarian checked for the absence of clinical signs in a herd concurrently at sampling times.

Follow-Up of M. bovis Infection. The 19 followup herds were initially sampled 4 times, at approximately 6-mo intervals, as we described in Vähänikkilä et al. (2019). Sampling was financially supported to motivate farmers to participate. Prioritizing youngest calves, nasal samples from a maximum of 20 calves (10-20) aged 1 wk to 9 mo were taken, including those kept at various locations. The sample size was set to include as many calves as possible in our herds. From most farms, there were 20 calves to sample between the ages 1 wk and 6 mo. In small herds with only a few calves, 10 was set as the accepted minimum and the age range was changed to 1 wk to 9 mo to get at least 10 samples. If there was a choice, animals with signs of a clinical disease were included in the sampling. In all farms but 1, all subclinical and clinical mastitis cases were tested using $M$. bovis PCR.

Thereafter, 10 herds voluntarily took nasal samples similarly from the calves to follow $M$. bovis infection status of the farms. The total number of samplings per herd ranged between 4 and 8 , and the duration of the sampling ranged from 18 mo to more than 5 yr (Figure $1)$. Most of the nasal swabs $(90 \%)$ were tested in the Finnish Food Authority (Kuopio, Finland) using a realtime PCR assay targeting the oppD gene of $M$. bovis (CFX96 Touch Real-Time PCR Detection System, Bio-Rad Laboratories, CA), as described in Sachse et al. (2010) with minor modifications described by Haapala et al. (2018). In 10 out of 101 sampling events, the NS samples were pooled and analyzed in a commercial nonaccredited laboratory Movet Oy (Kuopio, Finland). DNA extraction used an in-house method based on ultrahigh capacity DNA binding columns, and a commercial real-time PCR assay was used for detection of $M$. bovis (Thermo Fisher Scientific). The QMS and BTM samples were analyzed by commercial laboratories using a real time PCR assay for 16 mastitis pathogens, including $M$. bovis (Pathoproof Complete 16-kit, Thermo Fisher Scientific).

\section{Statistical Methods}

The data were entered into Excel spreadsheets (Microsoft Excel 2013, Microsoft Corp., Redmond, WA). Stata/MP version 16 (StataCorp, College Station, TX) was used for statistical analyses. External risk factors

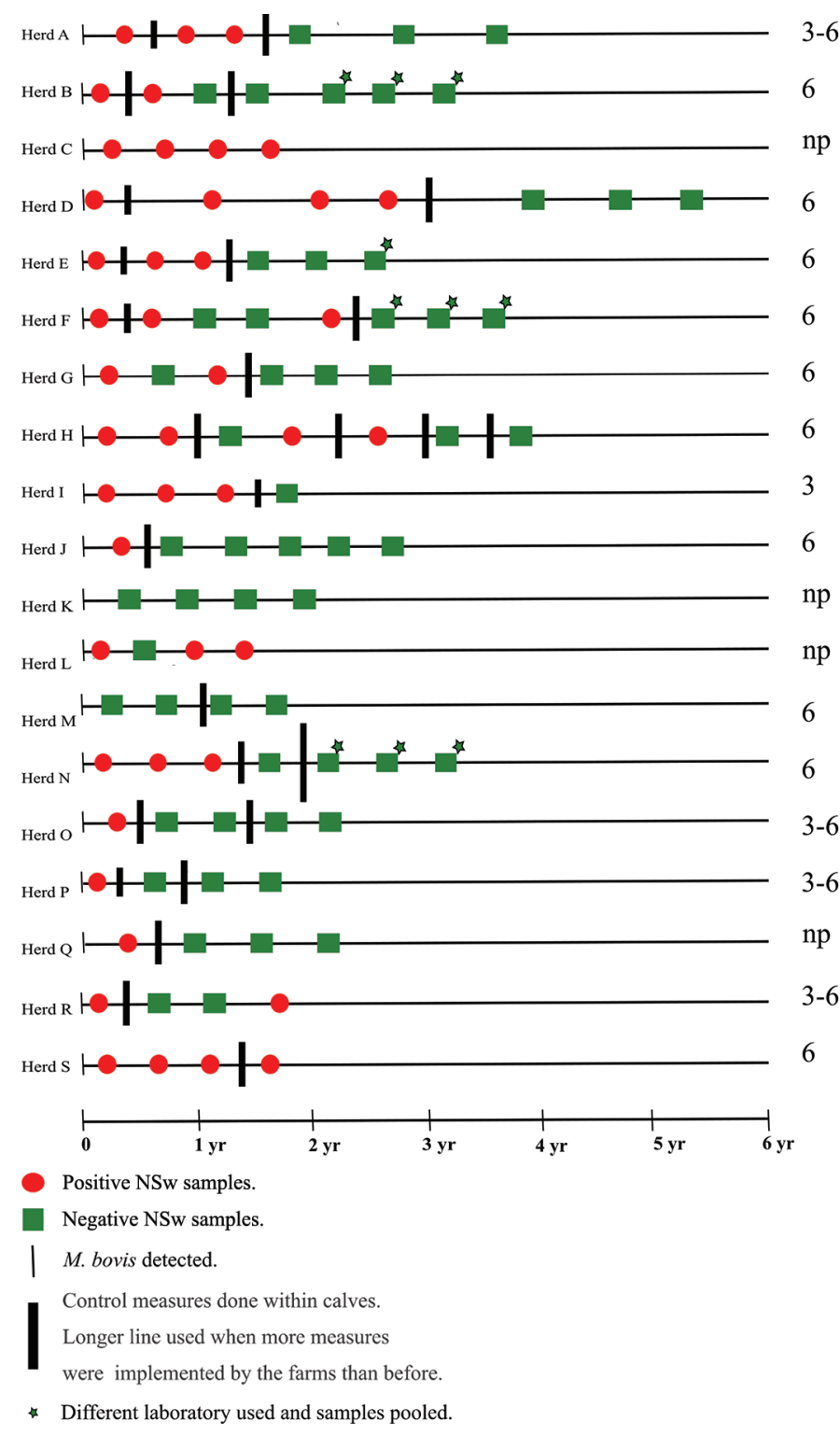

Figure 1. Timeline of the sampling for laboratory testing and control measures done for calves on the farms. A herd was considered positive during the visit if Mycoplasma bovis was detected with realtime PCR of nasal swabs (NSw). Description of the time of preventing nose-to-nose contact among the newborn calves: $\mathrm{np}=$ not prevented, $3=<3 \mathrm{mo}, 3-6=\geq 3$ and $<6 \mathrm{mo}, 6=\geq 6 \mathrm{mo}$. 
Table 3. Descriptive data and risk factors for the 40 case and 30 control farms with univariable analysis using Fisher's exact test and $t$-test $P$-values

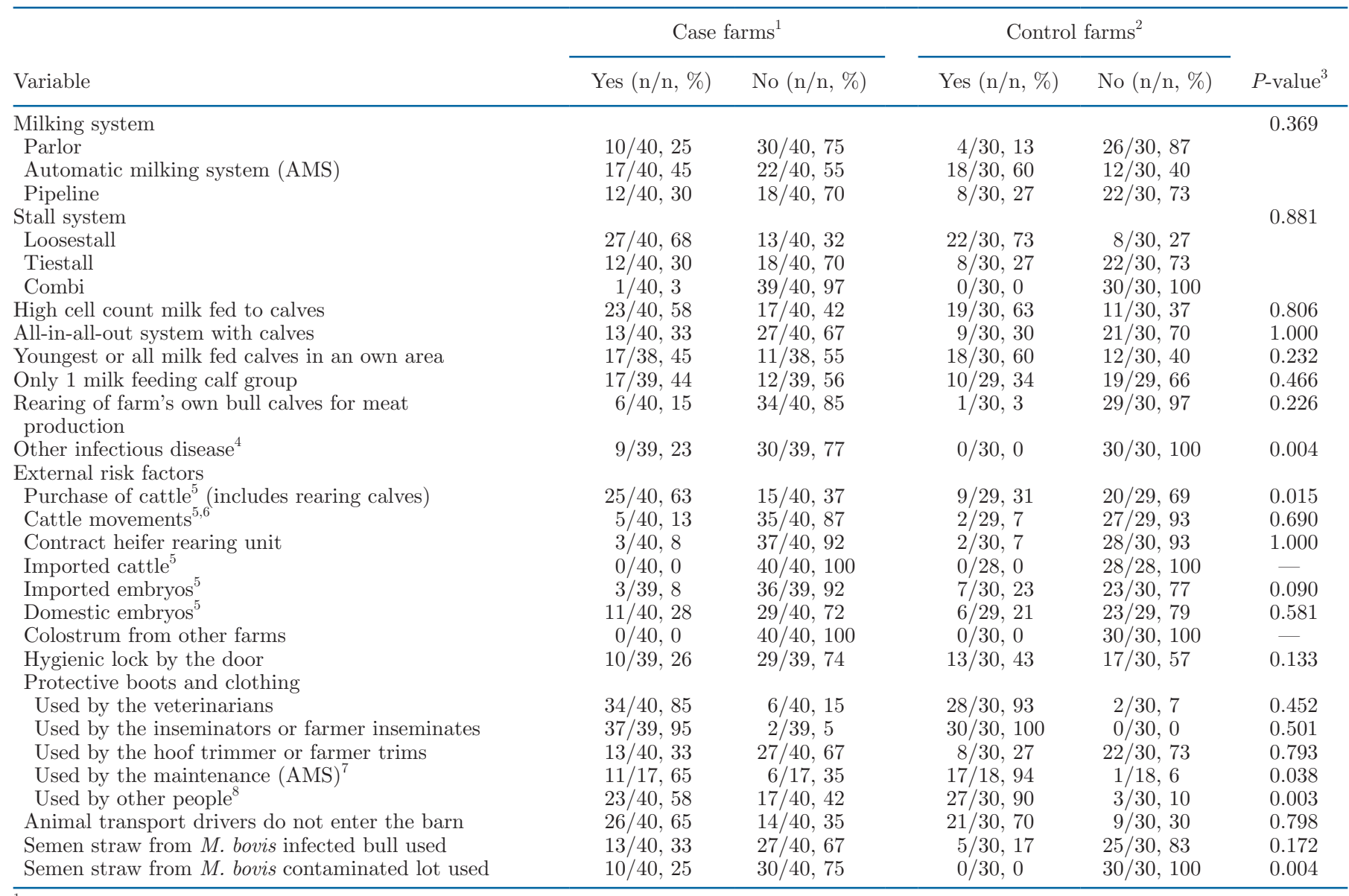

${ }^{1}$ Herd size median $=58$, range $=18-320, \mathrm{n}=40 ;$ herd average milk yield median $=9,681$, range $=5,537-11,922, \mathrm{n}=39$.

${ }^{2}$ Herd size median $=63$, range $=18-190, \mathrm{n}=30$; herd average milk yield median $=10,370$, range $=9,000-12,600, \mathrm{n}=30$.

${ }^{3}$ Case and control farms were matched to stall system, this often affecting the milking system and herd size. $P$-values for herd size and herd average milk yield were 0.355 and $<0.001$, respectively.

${ }^{4}$ Other infectious diseases within 6 mo affecting several animals (rotavirus, coccidiosis, interdigital phlegmon, respiratory disease, and Streptococcus agalactiae).

${ }^{5}$ Within 3 yr.

${ }^{6}$ Includes fairs $(\mathrm{n}=40)$, other farms, veterinarian's clinic, and so on $(\mathrm{n}=40)$ and other cattle visiting the farm $(\mathrm{n}=39)$.

${ }^{7}$ Third category being farms not using AMS (22 case farms and 12 control farms, one answer missing from case farms).

${ }^{8}$ Other visitors than in previous groups, for example, substitute farm workers.

for $M$. bovis infection were studied with a mixed effects logistic regression model. The outcome was negative or positive (no or yes) for farm level status of M. bovis infection. Positive farms had M. bovis bacteria detected, using either PCR or culture. External risk factors based on the questionnaire and farm visits were evaluated first with univariable analysis (Table 3).

Dichotomous (no or yes) variables were contract heifer rearing units, imported cattle, imported or domestic embryos used, colostrum from other farms used occasionally, hygienic lock by the door, and protective clothing and boots worn $(0=$ always, $1=$ not worn $)$ by veterinarians, inseminators, hoof trimmers, or other people. Additional dichotomous variables were animal transport drivers entering the barn and semen used in artificial insemination from one known $M$. bovisinfected bull or from particular M. bovis-contaminated lots from one bull (Lot POS). Categorical variable milking system [pipeline, parlor, or automated milking system (AMS)] was transformed to a dichotomous variable named AMS used (no or yes). Categories were also combined to form dichotomous variables related to purchased cattle and cattle movements. The variable purchasing cattle (no or yes) consisted of rearing 
calves bought from other dairy farms for meat production, in addition to purchasing breeding cattle. Cattle movements (no or yes) included combined questions on own cattle attending fairs or other places with cattle and other cattle visiting the farm. Protective clothing and boots worn by maintenance of AMS was included as a categorical variable $(0=$ used protective clothing and boots, $1=$ not used, $2=$ other than AMS milking system). Continuous variables were herd average milk yield and herd size (number of cows). The logarithmic transformation was used to normalize distribution with herd size.

Univariable analysis was done and the associations between the outcome and dichotomous variables were evaluated using Fishers exact test and the association between the outcome and the continuous variables were analyzed using Student's $t$-test. Herd size, AMS used, and milk yield were evaluated to be possible confounding factors with a causal diagram. Collinearity of variables was checked, and protective clothing and boots worn by maintenance of AMS were omitted due to correlation with variable AMS used.

External risk factors with $P<0.2$ in the univariable analysis and confounding factors were included in a final full logistic regression model. The final model consisted of the farm level status of infection as the outcome and predictors: artificial insemination from a known $M$. bovis infected bull, imported embryos, hygiene lock by the door, purchased cattle, protective clothing and boots used by other people, and confounding variables, herd average milk yield, AMS used, and herd size. Semen used from both a known $M$. bovis-infected bull and Lot POS had $P$-values $<0.2$, but semen used from a known $M$. bovis-infected bull was chosen because Lot POS was strongly unbalanced. All the positive lots were used in $M$. bovis-positive farms. Biologically meaningful interactions were tested for but were not evident. The predictors in the final nested model included artificial insemination from a known $M$. bovis infected bull, purchased cattle and confounding variables: herd average milk yield, AMS milking system and the herd size. The full and nested model did not differ regarding the likelihood ratio test. The model diagnostic was performed with Hosmer and Lemeshow's goodness-of-fit test and checking the residuals of the final nested model.

\section{RESULTS}

\section{Risk Factors}

Descriptive Data for the Farms and Internal Biosecurity Measures. The descriptive data and internal biosecurity measures for the case and control farms are presented in Table 3. The herd average milk yield was higher in the control than case herds $(P<$ 0.001). Infectious diseases other than $M$. bovis affecting several animals within 6 mo were more frequent in the case than control farms $(P=0.004)$. Other diseases reported by the farmers included ringworm, rotavirus, coccidiosis, interdigital phlegmon, respiratory disease, and Streptococcus agalactiae mastitis. Among the case farms, 2 herds had increased the herd size within the last year and 5 herds within the last 3 years. Among the control herds, 2 had increased the herd size in the last year and none within the last 3 years.

External Biosecurity Measures. The risk factors associated with the introduction of $M$. bovis infection on a farm are presented in Table 3. In univariable analysis, purchase of cattle $(P=0.015)$ and the use of semen from a known $M$. bovis-contaminated lot in insemination $(P=0.004)$ were more common on the case than on the control farms. In addition, protective clothing and footwear used among AMS maintenance staff and other people, including for example temporary help, were less used on the case than on the control farms. There was an association between herd average milk yield and the farm being $M$. bovis infected. In the final nested model, when the herd average milk yield decreased, the risk of a farm being $M$. bovis infected was lower. (Table 4). A slight trend can be seen in insemination with an M. bovis-positive bull (odds ratio $=3.7, P=0.096$ ) and in the herd size (odds ratio $=$ $3.4, P=0.065$; Table 4).

Table 4. Final model of the multivariable analysis of the external risk factors and confounding factors of farms becoming infected with Mycoplasma bovis

\begin{tabular}{|c|c|c|c|}
\hline Variable & $\mathrm{OR}^{1}$ & $P$-value & $95 \% \mathrm{CI}$ \\
\hline Semen straw from $M$. bovis infected bull used & 3.705 & 0.096 & $0.79-17.34$ \\
\hline Purchase of cattle & 2.980 & 0.103 & $0.80-11.06$ \\
\hline Automated milking system used & 0.340 & 0.167 & $0.07-1.57$ \\
\hline Herd average milk yield (per $100 \mathrm{~L}$ ) & 0.846 & 0.001 & $0.77-0.94$ \\
\hline Herd size $(\ln )^{2}$ & 3.364 & 0.065 & $0.92-14.42$ \\
\hline
\end{tabular}

${ }^{1}$ Odds ratio.

${ }^{2}$ Logarithmic transformation was used to normalize distribution. 


\section{Control Measures}

The farmers carried out the control measures in various ways that suited their individual possibilities and premise structures. Table 5 describes the measures that were carried out by the farmers at some point during the follow-up period. Figure 1 shows the timeline for the NS taken from calves and their PCR test results. In our study, 13 of 19 farms reached the low risk level. Ten herds, with a mean herd size of 103 , prevented nose-tonose contact (no contact) for at least 6 mo (Herds B, D, E, F, G, H, J, M, N, and S). Out of these herds, 5 kept the calves isolated for the first $3 \mathrm{mo}(\mathrm{D}, \mathrm{F}, \mathrm{G}, \mathrm{H}$, and $\mathrm{J})$. Out of these 10 herds, 8 herds reached the low risk level; Herds $\mathrm{H}$ and $\mathrm{S}$ did not. Four herds, with a mean herd size of 45, prevented nose-to-nose contact for 3 to 6 mo (Herds A, O, P, and R), and 1 of these isolated the calves for the first 3 mo (Herd R). Out of these 4 herds, 3 reached the low risk level (Herds A, O, P).
For 1 herd, calves were isolated or prevented from nose-to-nose contact for less than 3 mo (Herd I) and in 4 herds with a mean herd size of 70 , calves were not prevented from nose-to-nose contact (Herds C, K, L, and Q). Out of these farms, 2 reached the low risk level (Herds $\mathrm{K}$ and Q).

Sometimes the measures among calves were implemented gradually, as shown in Figure 1. All 19 farms culled the cows with $M$. bovis mastitis. Of those mastitic cows, $10 \%$ (5 of 51) had subclinical mastitis. All but 1 of the farms always had milk samples from cows with high SCC tested with $M$. bovis PCR; Herd E tested only occasionally.

The recommendation to move the calves away from the dam before the dam licked the newborn was followed consistently only on a few farms, so it was not evaluated in the presented control measures. Other recommendations given to the farmer and not included in the control measures were not recorded, but according

Table 5. Implementation of control measures on 19 follow-up farms $(1=$ yes, $0=$ no, np $=$ not prevented $)$. All farms culled the cows with subclinical or clinical Mycoplasma bovis mastitis. Control measures are described in Table 2

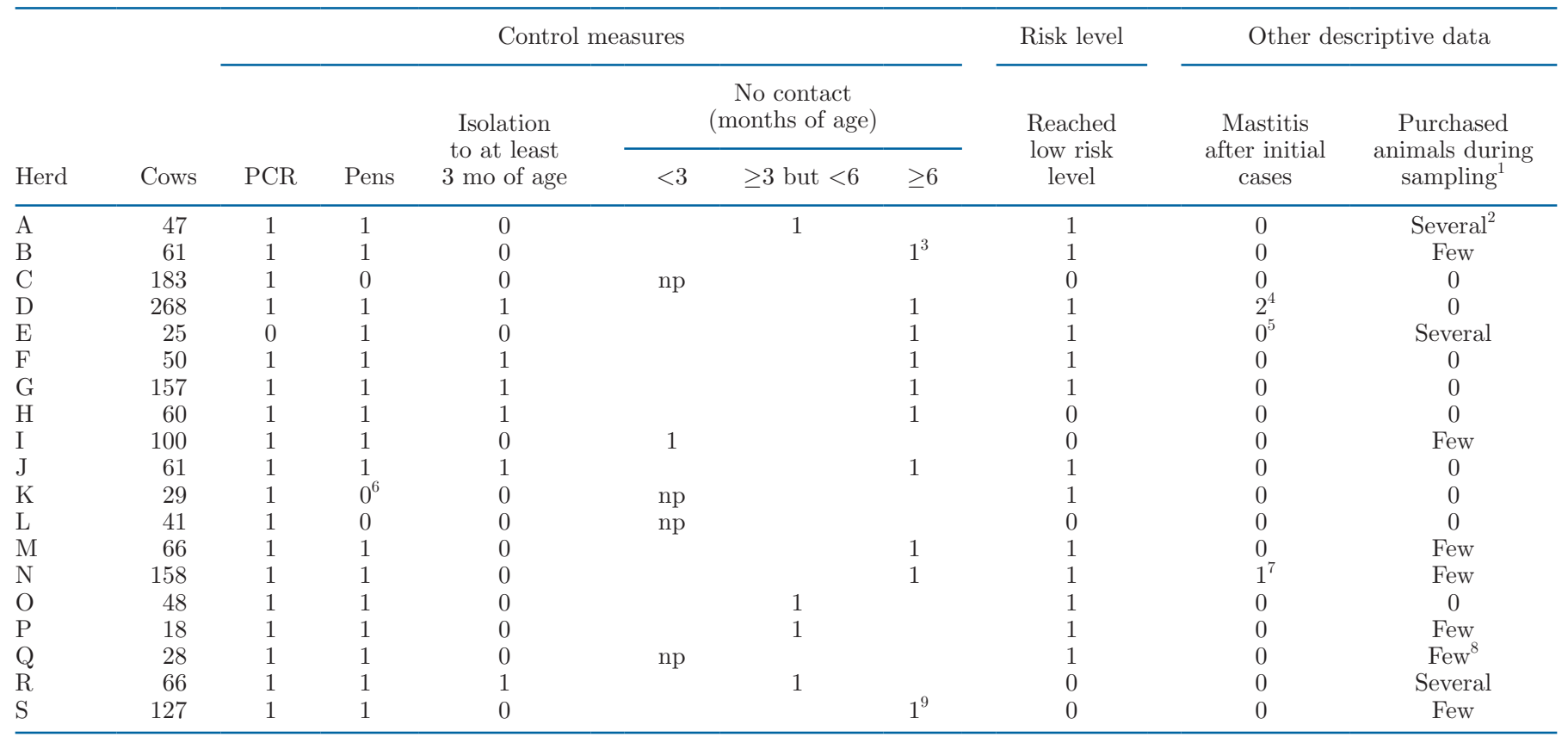

${ }^{1}$ Few $=1-8$ animals purchased, Several $=17-24$ animals purchased in addition to herd A, where 2 herds were joined.

${ }^{2}$ Three animals bought, in addition to merging a whole other herd.

${ }^{3}$ Nose-to-nose contact to cows occasionally might have been possible.

${ }^{4}$ Two mastitis cases after the initial outbreak, 1 more than 2 yr later and 1 about 3.5 yr later.

${ }^{5}$ One suspected case after about 6 mo due to antibodies in the bulk tank milk and individual milk samples; PCR was not done at drying off, later it was negative.

${ }^{6}$ The barn was washed and disinfected during the summer before the second sampling; sampling was done when the cattle were on pasture.

${ }^{7}$ One mastitis case 1 yr after the initial outbreak.

${ }^{8}$ Four Charolais cattle purchased but kept separate from other animals.

${ }^{9}$ Calves moved to corporate heifer unit at the age of 4 to 5 mo. 
to the discussions with the farmers were generally well followed, at least on many of the farms reaching low risk level.

\section{DISCUSSION}

There has been a drive to control $M$. bovis infection among dairy farms in Finland since the infection was first detected in the country in 2012. Animal Health ETT (Seinäjoki, Finland) has maintained a voluntary M. bovis control program since 2013. All farmers of the infected dairy herds were encouraged to control the infection and later join the program, in addition to being asked to consent to this study. The study included 40 recently discovered $M$. bovis-infected dairy herds that accounted for almost $70 \%$ of the infected farms at that time.

\section{Risk Factors}

We previously reported introduction of $M$. bovis into 2 naive herds by insemination with $M$. bovis-contaminated semen (Haapala et al., 2018). In this epidemiological study for risk factors, the case herds included a total of 10 farms that had used straws from the contaminated semen lots from the same $M$. bovis-infected bull. Semen from the same bull was used on both case and control farms, but semen straws from the known contaminated lots were only used on the case farms. For this reason, in our multivariable analysis, the use of $M$. bovis-contaminated semen lots could not be evaluated. A slight trend was seen in the risk of using the known $M$. bovis-positive bull in insemination. As only few of the lots tested positive (Haapala et al., 2018), the use of the bull does not measure the risk as accurately as if the contaminated semen lot were to have been used in the model. In the univariable analysis, the $P$-value was 0.004 for the contaminated semen lot used. Of the M. bovis-positive farms detected between 2012 and 2016 , about $25 \%$ of them had used contaminated semen lot from a particular bull and therefore could have obtained the infection from the semen. Although contamination of semen with $M$. bovis may occur only rarely, it might have an effect on the spread of the infection. However, the uneven presence of $M$. bovis in various lots and straws (Haapala et al., 2018) makes it very demanding to control the infection spread by overall testing of semen lots for $M$. bovis. On the other hand, testing lots seems useful, because control farms had not used contaminated lots.

Herd size was indicated to be a risk factor in the multivariable analysis, although it has to be regarded with caution as it could have been affected by the matching of the stall type between case and control farms. Similar results were found related to $M$. bovis or Mycoplasma sp. in other studies (Fox et al., 2003; Pinho et al., 2013; Murai and Higuchi, 2019). Murai and Higuchi (2019) reported that herds with more than 200 cows had higher odds for tank milk to be $M$. bovis positive. On the contrary, Gonzalez et al. (1992) reported that herd size was not a risk factor in the state of New York. Herds in Finland are small; the median herd size of the case and control herds was 58 and 63 respectively. Differences in these results can be due to herd size reflecting other risks that have not been evaluated in the studies.

Purchased animals were the third most important risk factor in univariable analysis. This factor was not significant in our multivariable analysis as in the results from other studies (Murai and Higuchi, 2019; Fujimoto et al., 2020). The prevalence of M. bovis among Finnish cattle is low, roughly $1 \%$ of the herds were infected in 2016 , and new M. bovis infections on dairy farms are efficiently detected through routine testing of mastitis cases with PCR detecting M. bovis. Furthermore, the cattle trade mostly occurs directly among few farms. This may explain why the risk from purchasing infected animals was low in our study.

Unlike in previous studies (Aebi et al., 2015), the herd average milk yield seemed to be a protective variable. In addition to small sample size, it can be associated with certain management practices on the farm; for example, farms with higher production are often better maintained and issues affecting the spread of infectious diseases might be better handled. In our study, the milk yield used was from a previous year before the infection was detected.

Farms with cattle infected with $M$. bovis experienced more infectious diseases in the previous 6 mo than the control farms, according to the results of univariable analysis. Occurrence of infectious diseases may be influenced by nondisease-specific risk factors, such as weaker external biosecurity, that expose a farm to $M$. bovis infection. Infectious diseases might also decrease the herd's resistance to other diseases.

\section{Control Measures}

We cannot fully analyze the efficacy of the control measures because it would have been unethical not to recommend any treatment of cattle on farms with an $M$. bovis infection. There were no farms that did not implement any of the advised measures and all the farms culled the $M$. bovis mastitic cows. However, there were farmers that decided not to adopt any of the recommended control measures regarding isolating the calves or avoiding nose contact with older animals. 
Young stock was not tested at the time of the study in the control farms; this may have resulted in some false negative herds in the control group.

The difficulties in detecting asymptomatic $M$. bovis positive animals are generally known. The fact that we took several NS samples consecutively increases the probability that the $M$. bovis-positive herds were detected at some point. In addition, our farms sampled mastitis routinely for $M$. bovis with PCR. Of the 19 follow-up farms included in this paper, $13(68 \%)$ reached the low risk status for $M$. bovis infection by January 2020. By the same time, $38 \%$ (33 of 86 ) of all the infected dairy farms recorded in the cattle herd health register of Animal Health ETT reached a low risk status. The figures are not directly comparable because most of the herds outside the study were infected later than the study herds, and there might not have been enough time to reach a low risk status. In 2015, approximately 150,000 samples from clinical and subclinical mastitis samples were analyzed with PCR detecting $M$. bovis, and there were about 285,000 cows in Finland. This intensive use of PCR to analyze bacteriological mastitis samples aids in detecting $M$. bovis mastitis cases quickly. The follow-up period was not of equal length in all farms and some farms started following the recommendations at the very end of the follow-up period. Some of these farms could have reached the low-risk level if they had followed the recommendation for a longer time.

The follow-up $M$. bovis infected farms culled both acute and subclinical $M$. bovis positive mastitic cows. This was possible because the numbers of $M$. bovis mastitic cows were not too high in any of the farms (Vähänikkilä et al., 2019). Byrne et al. (1998) reported on an $M$. bovis outbreak where the mastitis cases diminished within a few months as the infected cows were removed from the herd. However, Punyapornwithaya et al. (2012) reported that there was no evidence of an advantage in culling to control $M$. bovis mastitis. Neither of the studies mentioned taking samples from the young stock after the acute phase. The new mastitis cases after the initial cases or index case (Vähänikkilä et al., 2019) were on 2 farms that still clearly had $M$. bovis circulating among the calves and the remaining young stock.

In 4 herds, calves were not isolated or prevented from nose-to-nose contact. In spite of this, 2 of the farms (Herds K and Q) reached the low risk level. Both of the herds had fewer than 30 cows; for Herd K, the barn was washed and disinfected during the summer while animals were at pasture; for Herd Q, the calf pens were washed and disinfected. Low numbers of cows result in longer natural pauses in calving, which might break the chain of infection without the necessity of isolating calves or preventing nose-to-nose contact. For farms with more than 100 cows, it took a longer time to prepare new facilities for the calves, which might have prolonged the time needed to reach the low risk status.

Feeding of contaminated milk has been found to be a source of infection for calves (Butler et al., 2000). The fact that our farms were instructed not to give high SCC milk to the calves, that mastitis milk was tested with PCR, and that the farms culled all the detected $M$. bovis IMI cows probably lowered the risk for the isolated calves becoming infected through milk.

Surprisingly, avoiding nose-to-nose contact alone seemed to suffice in cases where isolation was insufficiently long, even though transmission by air should be possible in the same airspace. This is supported by Soehnlen et al. (2012), where M. bovis was not detected in the air in a veal barn with $M$. bovis-positive calves. In an older study, where agar plates were placed in a barn, bacterial growth of M. bovis was detected (Jasper et al., 1974). One explanation for these contradictory results might be differences in infection pressure and secretion of $M$. bovis. After an outbreak has passed, animals may secrete fewer bacteria, and the probability for air transmission might decrease.

$M$. bovis seems to spread quickly to all age groups as it enters the herd (Vähänikkilä et al., 2019). Young stock is considered to be the reservoir (Aebi et al., 2015), as the prevalence of $M$. bovis in the nasal samples among the calves can be high for several months (Bennett and Jasper, 1977). We presumed that the key to controlling $M$. bovis in dairy farms is to break the chain of infection with the calves. In our study, we implemented this among the calves born after the detection of M. bovis, by isolating and preventing nose-to-nose contact with older animals presumed to be infected with M. bovis.

More research is needed to determine precise times for isolation and prevention of nose-to-nose contact, because the control measures were implemented in various ways on the various farms. On each farm, control measures were aimed at breaking the chain of infection to the calves born after the detection of $M$. bovis on the farm. From what we learned during the study by comparing farms that managed to reach the low risk level with farms that did not, we recommend that the newborn calves should be isolated from infected cattle for at least 6 mo. In addition, nose-to-nose contact should be prevented with positive cattle at least until a year has passed from the start of the isolation, and ideally until the heifers join the milking cows. The first group of animals presumably free of $M$. bovis should always be moved to a pen that has been washed and disinfected.

If the older animals have not yet been cleared of the infection and secrete $M$. bovis, it might be possible for the heifers to become infected when they join the cows. This did not seem to be common in our study because 
so many farms reached the low risk level. Moreover, a calf positive at birth may infect the calves in the same pen. Keeping the groups separate and having an all-in-all-out raising system routinely helps to break the chain of infection. Calves that register positive at birth are hard to detect, but this did not seem to be a common occurrence in our study as so many farms had nasal smears from calves that registered negative for M. bovis. Effective routine testing of high SCC milk samples ( $>200,000$ cells $/ \mathrm{mL}$ ) using $M$. bovis PCR aids in early detection of $M$. bovis mastitis cases and $M$. bovis infected farms, thereby also helping to start control measures promptly.

\section{CONCLUSIONS}

Insemination with semen contaminated with $M$. bovis was significant in the univariable analysis but could not be fitted into a model as all positive lots were used in the case farms. An M. bovis positive bull used in insemination was placed in the model and a trend was seen in the risk. Thirteen out of 19 study herds most probably reached a low risk status for $M$. bovis infection without any positive samples in consecutive nasal samples by applying the advised control measures. The most important control measure is to isolate the calves or prevent nose-to-nose contact and to have routinely mastitic milk tested for M. bovis, in addition to culling $M$. bovis positive mastitis cows. A weakness of the study is that we did not have a clear control group for control measures. The control measures applied to calves are basic biosecurity procedures that help to control a wider range of infectious diseases. Better knowledge is required on the circulation and excretion of $M$. bovis in a herd, to be able to set more precise times for isolation and prevention of nose-to-nose contact.

\section{ACKNOWLEDGMENTS}

We acknowledge the Ministry of Agriculture and Forestry of Finland (Helsinki) for the main funding for this research project (grant number 1876/312/2013). We also acknowledge Valio Ltd. (Helsinki, Finland) for contributing to the funding and Doctoral Programme in Clinical Veterinary Medicine (University of Helsinki) for a funded doctoral candidate position, Faba for cooperation with collecting the data, and all the farms involved in the project. The authors declare that they have no conflicts of interest.

\section{REFERENCES}

Aebi, M., B. H. P. van den Borne, A. Raemy, A. Steiner, P. Pilo, and M. Bodmer. 2015. Mycoplasma bovis infections in Swiss dairy cattle: A clinical investigation. Acta Vet. Scand. 57:10. https://doi .org/10.1186/s13028-015-0099-x.

Bennett, R. H., and D. E. Jasper. 1977. Nasal prevalence of Mycoplasma bovis and IHA titers in young dairy animals. Cornell Vet. 67:361-373.

Bicknell, S.R., R. F. Gunning, G. Jackson, E. Boughton, and C. D. Wilson. 1983. Eradication of Mycoplasma bovis infection from a dairy herd in Great Britain. Vet. Rec. 112:294-297. https://doi .org/10.1136/vr.112.13.294.

Biosecurity New Zealand. 2020. Protection and response, Mycoplasma bovis. Accessed April 20, 2020. https://www.mpi.govt.nz/ protection-and-response/mycoplasma-bovis/.

Boddie, R. L., W. E. Owens, C. H. Ray, S. C. Nickerson, and N. T. Boddie. 2002. Germicidal activities of representatives of five different teat dip classes against three bovine mycoplasma species using a modified excised teat model. J. Dairy Sci. 85:1909-1912. https:/ /doi.org/10.3168/jds.S0022-0302(02)74266-5.

Brys, A., H. Pfützner, H. Bocklisch, and H. Weigel. 1992. Mycoplasma-bovis-freie aufzucht von rindern. (Mycoplasma bovis free breeding of cattle). Berl. Munch. Tierarztl. Wochenschr. 105:230-232.

Butler, J. A., S. A. Sickles, C. J. Johanns, and R. F. Rosenbusch. 2000. Pasteurization of discard mycoplasma mastitic milk used to feed calves: Thermal effects on various mycoplasma. J. Dairy Sci. 83:2285-2288. https://doi.org/10.3168/jds.S0022-0302(00)75114 $-9$.

Byrne, W. J., H. J. Ball, R. McCormack, and N. Brice. 1998. Elimination of Mycoplasma bovis mastitis from an Irish dairy herd. Vet. Rec. 142:516-517. https://doi.org/10.1136/vr.142.19.516.

Fox, L. K., D. D. Hancock, A. Mickelson, and A. Britten. 2003. Bulk tank milk analysis: Factors associated with appearance of Mycoplasma sp. in milk. J. Vet. Med. B Infect. Dis. Vet. Public Health 50:235-240. https://doi.org/10.1046/j.1439-0450.2003.00668.x.

Fujimoto, Y., H. Ito, H. Higuchi, H. Ohno, and K. Makita. 2020. A case-control study of herd- and cow-level risk factors associated with an outbreak of mycoplasma mastitis in Nemuro, Japan. Prev. Vet. Med. 177:104946. https://doi.org/10.1016/j.prevetmed.2020 .104946 .

Gautier-Bouchardon, A. V., S. Ferré, D. Le Grand, A. Paoli, E. Gay, and F. Poumarat. 2014. Overall decrease in the susceptibility of Mycoplasma bovis to antimicrobials over the past 30 years in France. PLoS One 9:e87672. https://doi.org/10.1371/journal.pone .0087672 .

Gille, L., J. Callens, K. Supré, F. Boyen, F. Haesebrouck, L. Van Driessche, K. van Leenen, P. Deprez, and B. Pardon. 2018. Use of a breeding bull and absence of a calving pen as risk factors for the presence of Mycoplasma bovis in dairy herds. J. Dairy Sci. 101:8284-8290. https://doi.org/10.3168/jds.2018-14940.

Gonzalez, R. N., P. M. Sears, R. A. Merrill, and G. L. Hayes. 1992. Mastitis due to Mycoplasma in the state of New York during the period 1972-1990. Cornell Vet. 82:29-40. https://doi.org/https:/ /hdl.handle.net/2027/coo.31924062891787?urlappend=\%3Bseq= 37.

Gorden, P. J., and P. Plummer. 2010. Control, management, and prevention of bovine respiratory disease in dairy calves and cows. Vet. Clin. North Am. Food Anim. Pract. 26:243-259. https://doi.org/ 10.1016/j.cvfa.2010.03.004.

Haapala, V., T. Pohjanvirta, N. Vähänikkilä, J. Halkilahti, H. Simonen, S. Pelkonen, T. Soveri, H. Simojoki, and T. Autio. 2018. Semen as a source of Mycoplasma bovis mastitis in dairy herds. Vet. Microbiol. 216:60-66. https://doi.org/10.1016/j.vetmic.2018 .02 .005 .

Hale, H. H., C. F. Helmboldt, W. N. Plastridge, and E. F. Stula. 1962. Bovine mastitis caused by a Mycoplasma species. Cornell Vet. 52:582-591.

Jasper, D. E., J. M. Al-Aubaidi, and J. Fabricant. 1974. Epidemiologic observations on mycoplasma mastitis. Cornell Vet. 64:407415. https://doi.org/https://babel.hathitrust.org/cgi/pt?id=uc1 .b4179400\&view $=1$ up\&seq $=423$.

Maunsell, F. P., A. R. Woolums, D. Francoz, R. F. Rosenbusch, D. L. Step, D. J. Wilson, and E. D. Janzen. 2011. Mycoplasma bovis 
infections in cattle. J. Vet. Intern. Med. 25:772-783. https://doi .org/10.1111/j.1939-1676.2011.0750.x.

Murai, K., and H. Higuchi. 2019. Prevalence and risk factors of Mycoplasma bovis infection in dairy farms in northern Japan. Res. Vet. Sci. 123:29-31. https://doi.org/10.1016/j.rvsc.2018.12.006.

Nicholas, R. A. J., and R. D. Ayling. 2003. Mycoplasma bovis: Disease, diagnosis, and control. Res. Vet. Sci. 74:105-112. https://doi.org/ 10.1016/S0034-5288(02)00155-8.

Pfützner, H., and K. Sachse. 1996. Mycoplasma bovis as an agent of mastitis, pneumonia, arthritis and genital disorders in cattle. Rev. Sci. Tech. 15:1477-1494.

Pinho, L., G. Thompson, M. Machado, and J. Carvalheira. 2013. Management practices associated with the bulk tank milk prevalence of Mycoplasma spp. in dairy herds in northwestern Portugal. Prev. Vet. Med. 108:21-27. https://doi.org/10.1016/j.prevetmed.2012.07 .001 .

Pothmann, H., J. Spergser, J. Elmer, I. Prunner, M. Iwersen, D. KleinJöbstl, and M. Drillich. 2015. Severe Mycoplasma bovis outbreak in an Austrian dairy herd. J. Vet. Diagn. Invest. 27:777-783. https:/ /doi.org/10.1177/1040638715603088.

Punyapornwithaya, V., L. K. Fox, D. D. Hancock, J. M. Gay, and J. R. Alldredge. 2012. Time to clearance of mycoplasma mastitis: The effect of management factors including milking time hygiene and preferential culling. Can. Vet. J. 53:1119-1122.

Sachse, K., H. S. H. Salam, R. Diller, E. Schubert, B. Hoffmann, and H. Hotzel. 2010. Use of a novel real-time PCR technique to monitor and quantitate Mycoplasma bovis infection in cattle herds with mastitis and respiratory disease. Vet. J. 186:299-303. https://doi .org/10.1016/j.tvjl.2009.10.008
Schibrowski, M. L., J. S. Gibson, K. E. Hay, T. J. Mahony, and T. S. Barnes. 2018. Mycoplasma bovis and bovine respiratory disease: A risk factor study in Australian feeder cattle. Prev. Vet. Med. 157:152-161. https://doi.org/10.1016/j.prevetmed.2018.06.005.

Soehnlen, M. K., A. Aydin, K. S. Murthy, E. J. Lengerich, A. L. Hattel, B. A. Houser, G. D. Fenton, H. R. Lysczek, C. M. Burns, A. M. Townsend, J. W. Brooks, D. R. Wolfgang, and B. M. Jayarao. 2012. Epidemiology of Mycoplasma bovis in Pennsylvania veal calves. J. Dairy Sci. 95:247-254. https://doi.org/10.3168/jds.2011 -4309 .

Timsit, E., M. Arcangioli, N. Bareille, H. Seegers, and S. Assié. 2012. Transmission dynamics of Mycoplasma bovis in newly received beef bulls at fattening operations. J. Vet. Diagn. Invest. 24:1172-1176. https://doi.org/10.1177/1040638712463211.

Vähänikkilä, N., T. Pohjanvirta, V. Haapala, H. Simojoki, T. Soveri, G. F. Browning, S. Pelkonen, N. K. Wawegama, and T. Autio. 2019. Characterisation of the course of Mycoplasma bovis infection in naturally infected dairy herds. Vet Microbiol. 231:107-115. https://doi.org/10.1016/j.vetmic.2019.03.007.

\section{ORCIDS}

Vera Haapala (ㄴ) https://orcid.org/0000-0002-2482-3090

Nella Vähänikkilä @ https://orcid.org/0000-0002-5164-9059

Tiina Autio @ https://orcid.org/0000-0001-8040-984X

Heli Simojoki ๑ https://orcid.org/0000-0002-4095-4461 